\title{
Constraining the solutions of an inverse method of stellar population synthesis ${ }^{\star}$
}

\author{
J. Moultaka ${ }^{1,2}$, C. Boisson ${ }^{2}$, M. Joly ${ }^{2}$, and D. Pelat ${ }^{2}$ \\ 1 Physikalisches Institut, Zülpicher Str. 77, 50937 Köln, Germany \\ ${ }^{2}$ LUTH, Observatoire de Meudon, 5 place Jules Janssen, 92190 Meudon Cedex, France
}

Received 19 September 2003 / Accepted 10 February 2004

\begin{abstract}
In three previous papers (Pelat 1997, MNRAS, 284, 365; Pelat 1998, MNRAS, 299, 877; Moultaka \& Pelat 2000, MNRAS, 314, 409), we set out an inverse stellar population synthesis method that uses a database of stellar spectra. Unlike other methods, this one provides full knowledge of all possible solutions as well as a good estimation of their stability; moreover, it provides the unique approximate solution, when the problem is overdetermined, using a rigorous minimization procedure. In Boisson et al. (2000, A\&A, 357, 850), this method was applied to 10 active and 2 normal galaxies. In this paper we analyse the results of the method after constraining the solutions. Adding a priori physical conditions to the solutions constitutes a good way to regularize the synthesis problem. As an illustration we introduce physical constraints on the relative number of stars taking into account our present knowledge of the initial mass function in galaxies. To avoid biases on the solutions due to such constraints, we use constraints involving only inequalities between the number of stars, after dividing the H-R diagram into various groups of stellar masses. We discuss the results for a well-known globular cluster of the galaxy M 31 and discuss some of the galaxies studied in Boisson et al. (2000, A\&A, 357, 850). We find that, given the spectral resolution and the spectral domain, the method is very stable according to such constraints (i.e. the constrained solutions are almost the same as the unconstrained one). However, additional information can be derived about the evolutionary stage of the last burst of star formation, but the precise age of this particular burst seems to be questionable.
\end{abstract}

Key words. galaxies: stellar content - galaxies: active - methods: analytical

\section{Introduction}

The search of the stellar populations inside unresolved galaxies has been the aim of several studies since the 1960s. Two different approaches have been adopted for this purpose: the direct approach of which methods are usually called "the evolutive synthesis methods" (e.g. Tinsley 1972; Charlot \& Bruzual 1991; Bruzual \& Charlot 1993; Leitherer et al. 1999; Fioc \& Rocca-Volmerange 1997; Vazdekis 1999; Bruzual \& Charlot 2003) and the inverse ones called "the synthesis methods" (e.g. Faber 1972; Joly 1974; O’Connell 1976; Bica 1988; Schmidt et al. 1989; Silva 1991; Pelat 1997, 1998; Moultaka \& Pelat 2000).

In the first approach, one decides an a priori model for the history of the star formation occuring inside the studied galaxy, and by means of theoretical stellar evolutionary tracks and of a stellar database derives quantities that are directly compared to the observed ones. From different input models, one retains the model that best fits the observed quantities.

Send offprint requests to: J. Moultaka,

e-mail: moultaka@ph1.uni-koeln.de

* Appendix A, Figs. 2-5 and Tables 4-6 are only available in electronique form at http://www. edpsciences.org
In the inverse approach, usually, no a priori model is necessary to derive the stellar populations and one uses exclusively the observables to deduce the stellar spectral types and luminosity classes by means of a minimization procedure. This minimization task is not a very simple one since the absolute minimum is usually difficult to find because the "objective function" (which is the function that one has to minimize) can rarely be minimized analytically.

Whatever the approach, the problem of stellar population synthesis often suffers from the lack of true solutions or, on the contrary, from their degeneracy (i.e. multiple solutions) and/or from their instability (i.e. small errors around the observations can induce discontinuities in the solutions). These three inconveniences have been controlled in the inverse method described in Pelat (1997, 1998, hereafter Papers I and II) where all the solutions are well identified and the minimization procedure is rigorously treated, as well as in Moultaka \& Pelat (2000) where the stability of the various solutions is analysed (hereafter Paper III).

In this paper, we study the influence on the different solutions of astrophysical constraints included a priori when searching for a solution. The concept of constraining the solutions in the inverse methods has been adopted by 
O'connell (1976), Pickles (1985) and Silva (1991) but no estimation of the induced bias has been given by these authors to our knowledge.

In the next section, we recall briefly the inverse method and its error analysis; in the third section, we describe constrained models. In Sect. 4, we show and discuss constrained versus unconstrained results for the globular cluster G170 located in M 31, the LINER NGC 4278, the starburst NGC 3310 and the Seyfert 2 galaxy NGC 2110. In the last section we give a general description of the behaviour of constrained solutions in the 27 central regions of the twelve galaxies studied in Boisson et al. (2000), hereafter Paper IV.

\section{Description of the inverse method}

As described in Pelat $(1997,1998)$, the present inverse method uses the equivalent widths of galactic spectra absorption lines as observables to be fitted by a combination of the continuum fluxes and equivalent widths of a stellar line database. The basic equation providing the synthetic equivalent widths is:

$W_{\mathrm{syn} j}=\frac{\sum_{i=1}^{n_{\star}} k_{\lambda_{0} i} W_{j i} I_{j i}}{\sum_{i=1}^{n_{\star}} k_{\lambda_{0} i} I_{j i}} \quad$ for $j=1, \ldots, n_{\lambda}$

where $W_{\mathrm{syn} j}$ is the synthetic equivalent width of a line at wavelength $\lambda_{j}, W_{j i}$ and $I_{j i}$ are respectively, the equivalent widths and continuum fluxes (normalized at a reference wavelength $\lambda_{0}$ ) of the same lines measured in stars of class $i ; k_{i}$ is the contribution of star class $i$ to luminosity at the reference wavelength $\lambda_{0} ; n_{\star}$ and $n_{\lambda}$ are the total number of stars considered in the database and the total number of lines measured in the spectra.

To this equation, we add two physical conditions, that all the stellar contributions to luminosity $k_{i}$ are positive and their sum equals one:

$k \geq 0$

$\sum_{i=1}^{n_{\star}} k_{i}=1$

Thus, having the observed set of galactic equivalent widths $W_{\mathrm{obs} j}$, one searchs for the stellar contributions $k_{i}$ satisfying the previous physical conditions and minimizing the following objective function which is the square of what is called the synthetic distance $D$. The synthetic distance represents a kind of $\chi^{2}$ considering the $W_{\mathrm{obs} j}$ as data. In fact $D^{2}$ would be exactly a $\chi^{2}$ if $P_{j}^{-1}$ were chosen as the $W_{\mathrm{obs} j}$ variances (i.e. $P_{j}=\sigma_{W_{\mathrm{obs} j} ;}^{-2}$, see Sect. 5 of Paper I for a discussion on the $\sigma_{W_{\text {obs } j}}$ value):

$D^{2}=\sum_{j=1}^{n_{\lambda}}\left(W_{\mathrm{obs} j}-W_{\mathrm{syn} j}\right)^{2} P_{j}, P_{j} \geq 0$.

In this definition, $P_{j}$ is a weight characterizing the quality of the equivalent width measurements. One can eliminate this weight by applying the change of variables $W_{j} \leftrightarrow W_{j} \sqrt{P}_{j}$. In the following, we will consider this change of variables as already made. As stated, the problem can either be overdetermined (i.e. there are more equations than unknowns) or underdetermined (i.e. there are more unknowns than equations). In the first case, one finds at most a unique solution and, because of observational errors, there is usually not an exact solution; then the adopted solution is the approximate one which minimizes the synthetic distance. In Paper I, it is shown that this minimum is unique and near the true one; in addition, it is demonstrated that when the signal to noise ratio goes to infinity, the unique minimum is exactly the true one (see Paper I).

In the underdetermined case, one gets an infinite number of solutions that are convex combinations of particular solutions called the extreme solutions (see Paper II).

The search of the error regions around the solutions was made in Paper III, giving thus the relevance of the solutions and a criterium to sort the various solutions in the underdetermined case by order of merit. An "ideal" database (i.e. a database with an infinite spectral resolution, the largest wavelength domain and adapted for the velocity dispersion observed in the studied galaxy) is not degenerate. Then the analysis made in Paper III gives equivalently a condition to the database (depending on the quality of the observations) allowing one to get a welldefined solution. Depending on the quality of the observations (i.e. on the size of the galactic error region), the stellar database may become degenerate. As the $\mathrm{S} / \mathrm{N}$ ratios of the stellar spectra are usually higher than those of the galaxy, more than one star may lie inside the galactic error region in the equivalent width vector space. This situation leads to very poorly determined solutions, because the different stars cannot all be distinguished in this case. One has to eliminate such correlated stars from the database to obtain a well-defined solution. In the Appendix, the computation of the standard deviation around the synthetic distance is described.

\section{The constraints model}

\subsection{The stellar database}

The database used comes from three different stellar libraries: Serote Roos et al. (1996), Silva \& Cornell (1992) and Fluks et al. (1994). The spectral resolution of the resulting stellar database is $11 \AA$ and the spectral domain goes from $5000 \AA$ to $8800 \AA$ A. Given the galaxy velocity dispersion, the velocity broadening of the lines is equivalent to the spectral resolution of the stellar database. Thus no correction for velocity dispersion has to be applied. In this domain and at this spectral resolution, we have selected 47 line features and measured their equivalent widths (see Paper IV); some wavelength intervals have not been included in the database because of atmospheric absorption bands not corrected in the Silva \& Cornell library. The table of the line intervals is shown in Boisson et al. (2000). Stars included in the database have been chosen so that the $\mathrm{H}-\mathrm{R}$ diagram is best represented in spectral types and luminosity classes for two metallicities (solar and about twice solar).

\subsection{The model}

In general, the synthesis problem is what is called in mathematical terms an "Ill-posed" problem. This means that the problem may have no solution or a large number of solutions and/or 
that the solution is not stable against small deviations around the observation. The usual way to overcome this difficulty is to regularize the problem. This can be done by searching the solution of maximal entropy for example or using any other reasonable criterium. We suggest here to regularize the problem using physical criteria to obtain a unique and stable solution. This regularization procedure has already been adopted in previous papers when the positivity conditions $\left(k_{i} \geq 0\right)$ were considered. In the present paper, we introduce more physical conditions to constrain the solution.

Technically, constraining the solutions of an inverse problem reduces the volume of the simplex of solutions or the synthetic surface as defined in Paper I (which is the set of exact solutions). The problem with such a procedure is that the solution may be biased by the constraints model while, according to the philosophy of the inverse approach, it should not be altered by any a priori overconstrained model (otherwise it will reflect the model itself and will not provide additional information on the real stellar population). This inconvenience can be verified if the solutions are found to be on the border of the simplex (where this one has been reduced), because in this case, it would mean that the solution has been strongly constrained to enter in the reduced simplex (otherwise, it would lie inside or on the other borders of the simplex).

We can summarize the search for a solution as:

having the data (the spectra), one uses a model to derive the stellar contributions to luminosity $k_{i}$; this is the inverse approach. This process could, as shown in Paper II, provide an infinite number of exact solutions (in the underdetermined case) or an approximate solution obtained by a minimization procedure (in the overdetermined case). The obtained solutions are "mathematical" solutions which solve rigourously the mathematical problem. They could be stable or unstable to small deviations around the observation. To reduce the number of solutions and to ensure that the solutions satisfy the physical conditions of the studied object, it is necessary to constrain the problem.

Thus, the synthesis problem may be stated as minimizing the synthetic distance of Eq. (4) also written in the following form:

$D^{2}=\sum_{j=1}^{n_{\lambda}}\left(\frac{\sum_{i=1}^{n_{\star}}\left(W_{\mathrm{obs} j}-W_{j i}\right) I_{j i} k_{i}}{\sum_{i=1}^{n_{\star}} I_{j i} k_{i}}\right)^{2}$

(where the change of variable $W_{j} \leftrightarrow W_{j} \sqrt{P}_{j}$ has been applied). The set of stellar contributions $\boldsymbol{k}=\left(k_{1}, k_{2}, \ldots k_{n_{\star}}\right)$ is submitted to the conditions:

$\boldsymbol{l} \leq\left\{\begin{array}{c}\boldsymbol{k} \\ \mathbf{C} \boldsymbol{k}\end{array}\right\} \leq \boldsymbol{u}$.

In the previous inequalities, $\boldsymbol{l}$ and $\boldsymbol{u}$ are the lower and upper constant limits and the first inequality takes into account that all contributions are positive and less than one because of condition (3), therefore the first $n_{\star}$ components of vectors $\boldsymbol{l}$ and $\boldsymbol{u}$ are equal to zero and one. $\mathbf{C}$ is the constraint matrix of $n_{\star}$ columns in which the first line is a vector of components equal to one to express constraint number (3). The component number $n_{\star}+1$ of vectors $\boldsymbol{l}$ and $\boldsymbol{u}$ is equal to one.

Since the expression of the synthetic distance of Eq. (5) is not linear in $\boldsymbol{k}$, we decided to minimize instead the linear function $\|\mathbf{A} \boldsymbol{k}\|^{2}$ submitted to the above constraints (where A is a matrix defined in Paper I of whose components are $\left.A_{j i}=\left(W_{\mathrm{obs} j}-W_{j i}\right) I_{j i}\right)$. This function happens to be the "principal synthetic distance" defined in Paper I as:

$D_{\text {prin }}^{2}=\sum_{j=1}^{n_{\lambda}}\left(W_{\mathrm{obs} j}-W_{\mathrm{syn} j}\right)^{2} I_{\mathrm{syn} j}^{2}, \quad I_{\mathrm{syn} j}=\sum_{i=1}^{n_{\star}} I_{j i} k_{i}$.

As it is stated in Paper I, the problem then possesses a unique solution. Thus, using the constrained least square method, we can derive the unique set of stellar contributions minimizing the principal synthetic distance and satisfying the model constraints (6). Once the principal solution is at hand, we can find the "principal geometrical" one which is the nearest solution to the constrained principal solution and minimizes the initial synthetic distance of Eq. (5). For this purpose, we use the same iterative method as in Paper I where the iteration is made on the synthetic surface. At each step of iteration $(m+1)$ and using the constrained least square method, we search for the new solution $\boldsymbol{k}^{(m+1)}$ that minimises the following function:

$$
\left\|\mathbf{X}^{(m+1)} \boldsymbol{k}^{(m+1)}-\boldsymbol{b}\right\|^{2}
$$

submitted to the constraints of Eq. (6) and where $\boldsymbol{b}$ is defined by the relation $\boldsymbol{b}=\boldsymbol{W}_{\mathrm{obs}}-\boldsymbol{W}_{\text {syn }}+\mathbf{X} \boldsymbol{k}^{(m)}$.

The solution of the constrained least square method is obtained using procedures from the NAG library.

\subsection{The constraints}

As the spectral resolution and the wavelength range are limited, the number of uncorrelated stars is also limited. This leads to inherent incompleteness of the database which together with different photometric accuracy between galactic and stellar spectra can lead to non-physical solutions. So, one may have to constrain the solutions, in particular in such a way that the Initial Mass function of stars (IMF) satisfies the known shape of this function derived from observations of resolved objects.

We first define groups of main sequence (MS) stars following their range of lifetimes. This translates into stellar mass intervals. In each of these groups, we include the evolved stars of the same initial mass. Tables 1 and 2 show the sorting of the stellar database in "mass groups" for two different mass resolutions. The mass interval associated with each star of the database was determined using Schmidt-Kaler tables (1982). When necessary, we interpolated the available masses in the tables. Concerning the evolved stars, we used the evolutionary tracks of the Padova and Geneva groups to associate an interval of initial masses for the stars. Each star thus represents an evolutionary stage in a given mass group. We aim not to privilege one of the classical IMFs (as the functions of Salpeter 1955; Scalo 1986; Kroupa et al. 1993). 
Table 1. The mass groups sorting of the stellar database in the case of the "Standard mode".

\begin{tabular}{ccc}
\hline \hline Mass group & Mass interval & Stars \\
\hline 1 & $17 M_{\odot}-30 M_{\odot}$ & O7-B0V \\
& M2Ia \\
\hline 2 & $3 M_{\odot}-17 M_{\odot}$ & B3-4V \\
& & G0Iab, K4Iab, rG2Iab, rK0II, rK3Iab \\
\hline 3 & $1.6 M_{\odot}-3 M_{\odot}$ & A1-3V \\
\hline 4 & $0.8 M_{\odot}-1.6 M_{\odot}$ & F2V, F8-9V, G4V, rG0IV, rG5IV \\
& & G0-4III, wG8III, G9III, K4III, M0.5III, M4III, M5III, rG9III, rK3III, rK3III(bis), rK5III \\
\hline 5 & $\leq 0.8 M_{\odot}$ & G9-K0V, K5V, M2V, rK0V, rK3V, rM1V \\
\hline
\end{tabular}

Table 2. The mass groups sorting of the stellar database in the case of the "Decreasing IMF mode".

\begin{tabular}{ccc}
\hline \hline Mass group & Mass interval & Stars \\
\hline 1 & $17 M_{\odot}-30 M_{\odot}$ & O7-B0V \\
& & M2Ia \\
\hline 2 & $3 M_{\odot}-17 M_{\odot}$ & B3-4V \\
& & G0Iab, K4Iab, rG2Iab, rK0II, rK3Iab \\
\hline 3 & $1.6 M_{\odot}-3 M_{\odot}$ & A1-3V \\
\hline 4 & $1.4 M_{\odot}-1.6 M_{\odot}$ & F2V \\
& & M4III, M5III \\
\hline 5 & $1.1 M_{\odot}-1.4 M_{\odot}$ & F8-9V, rG0IV, rG5IV \\
& & G0-4III, wG8III, G9III, K4III, M 0.5III, rG9III, rK3III, rK3III(bis), rK5III \\
\hline 6 & $0.8 M_{\odot}-1.1 M_{\odot}$ & G4V \\
\hline 7 & $0.7 M_{\odot}-0.8 M_{\odot}$ & G9-K0V, rK0V, rK3V \\
\hline 8 & $0.5 M_{\odot}-0.7 M_{\odot}$ & K5V \\
\hline 9 & $\leq 0.5 M_{\odot}$ & M2V, rM1V
\end{tabular}

We will discuss here two different modes of constraints corresponding to the two different samplings in mass of the H-R diagram shown in Tables 1 and 2:

\section{The "Standard mode"}

In this mode, we impose a general constraint on the number of born stars of the different mass groups:

$N_{i} \leq N_{j}$

where $N_{i}$ and $N_{j}$ are respectively the numbers of born stars with masses located in the mass intervals $] M_{i a}, M_{i b}[$ and $] M_{j a}, M_{j b}$ [ and where $M_{j b} \leq M_{i a}$. Even though a galaxy is not described by a single burst of star formation, Eq. (9) is valid as well for all the Main Sequence (MS) stars produced by a continuous star formation scenario or by multiple bursts of star formation. As stars of higher masses evolve faster than the ones of smaller masses, Eq. (9) is also valid for all stars (MS and evolved) belonging to the same mass groups.

This translates into the following constraint set:

$N_{\mathrm{MS} i} \leq N_{\mathrm{MS} j}$

$N_{\mathrm{T} i} \leq N_{\mathrm{T} j}$

where $N_{\mathrm{MS} i}$ and $N_{\mathrm{T} i}$ are the number of MS stars and the total number of stars (MS and evolved stars) in a given mass interval. In Table 1, the mass intervals are chosen to be the narrowest possible such that Eqs. (10) and (11) are satisfied in the case of the classical IMFs.

A consequence of introducing such constraints is that the resulting solutions will present higher synthetic distances than in the unconstrained solution, and therefore the fit will be less good.

\section{The "Decreasing IMF mode"}

The second mode of constraints called "the Decreasing IMF mode" states that all initial mass functions are decreasing functions. Consequently, for a burst of star formation, the total number of born stars $N_{\mathrm{T} i}$ of a given mass group $\left(N_{\mathrm{T} i}=\right.$ $N_{\mathrm{MS} i}+N_{\text {evolved } i}$ ) of a given group of stars corresponding to a mass interval $] M_{i a}, M_{i b}\left[\right.$ with a mean mass $M_{i}\left(M_{i}=\frac{M_{i a}+M_{i b}}{2}\right)$ is such that:

$N_{\mathrm{T} i} \leq \frac{\Delta \log M_{i} * N_{\mathrm{T} j}}{\Delta \log M_{j}}$

where $M_{j}<M_{i}$.

$\Delta \log M_{i}$ and $\Delta \log M_{j}$ are respectively the logarithmic lengths of each mass interval. The difference with respect to Eq. (8) is that the number of stars in each group is weighted by the mass.

As in the "Standard mode", Eq. (12) is valid for the number of MS stars on the one hand and the total number (MS and evolved) stars on the other hand. Then, one can write:

$$
\begin{aligned}
& N_{\mathrm{MS} i} \leq \frac{\Delta \log M_{i} * N_{\mathrm{MS} j}}{\Delta \log M_{j}} \\
& N_{\mathrm{T} i} \leq \frac{\Delta \log M_{i} * N_{\mathrm{T} j}}{\Delta \log M_{j}}
\end{aligned}
$$

where the notations are the same as for the "Standard mode". 
As Eq. (12) depends on the mass intervals, the resulting mass sorting is more refined for the low mass stars (see Table 2). Because of the better resolution of the mass sorting in this case, the number of constraints is increased compared to the "Standard mode". Consequently, the synthetic distance in this mode of constraints will be higher.

The previous description of the adopted constraints shows that these take the form of large inequalities between the numbers of stars or equivalently, in the optical domain, between the stellar contributions to luminosity at the reference wavelength $k_{\lambda_{0 i}}$. The relation between the number of stars of class $i$ and their contribution to luminosity is:

$n_{i}=\frac{k_{\lambda_{0 i}} L_{\lambda_{0} \mathrm{gal}}}{l_{\lambda_{0 i}}}$

where $L_{\lambda_{0} \text { gal }}$ is the galactic (or the cluster) luminosity at the reference wavelength $\lambda_{0}$ and $l_{\lambda_{0 i}}$ is the luminosity of a star of class $i$ at the same wavelength.

As the constraints have the form of large inequalities (i.e. equalities are allowed) one may obtain solutions on the border of the domain of constraints (i.e. if the constraints are satisfied with equalities), the solution is probably over-constrained. In such a case, one has to conclude that the constraint set is not "appropriate" for the data.

\section{Results}

The effect of adding constraints to the inverse method was tested on the globular cluster G170 located in M 31 and three central regions of galaxies corresponding to three types of galaxy activity considered in Paper IV.

As the aim of the present paper is to study the effects of constraining the solutions, we will only concentrate on a description and on the comparison of the different solutions. Therefore no complete physical discussion (especially in the case of the galaxies) will be made in the following sections.

In Tables 3 to 6 we list the stars present in the database (Col. 1), the unconstrained solution (only statisfying the positivity condition (2) and Eq. (3)) in Col. 2. In the two following columns, we show the mass intervals corresponding to the mass sorting for the "Decreasing IMF mode" and the constrained solutions of this mode. Columns 5 and 6 exhibit the mass intervals and the constrained solutions in the case of the "Standard mode". Finally, in some examples, we show particular solutions where we imposed a fixed value to one or more stellar contributions.

The solutions list the stellar contributions, $\boldsymbol{k}_{i}$, to luminosity at the reference wavelength $\lambda_{0}=5450 \AA$.

\subsection{The stellar cluster $\mathrm{G} 170$}

To validate our method, we compare the constrained and unconstrained solutions obtained for a single stellar population system, namely, the well-studied globular cluster G170 located in the galaxy M 31. The globular cluster spectrum is taken from Jablonka et al. (1992).

The unconstrained solution (see Table 3 ) shows that this cluster has a solar metallicity. The turnoff in G4V suggests an age of about $10^{10}$ years and the contribution of dwarf stars to the luminosity of $57 \%$ shows that the luminosity in the optical is dominated by the MS stars. The metallicity is clearly solar (the contribution of stars of solar metallicity is about $\sim 92 \%$ ). The measured internal reddening $E(B-V)$ is of $\sim 0.05$. This value of the reddening is determined as the correction to be applied to the observed spectrum to match the synthetic one. In this process the Galactic law is parametrized as in Howarth (1983).

These results agree well with those found by Jablonka et al. (1992); the authors concluded, using measurements of equivalent widths of absorption lines, that this cluster has a solar metallicity as well as an age of $2 \times 10^{10}$ years.

The "Decreasing IMF mode" presents a solution with acceptable synthetic distance in the sense that its value is at $\sim 1 \sigma$ from the synthetic distance value of the unconstrained solution, but the contributions are slightly different: only $\sim 38 \%$ of the optical luminosity is due to MS stars and $\sim 20 \%$ of it is due to metallic stars.

The constrained solution of the "Standard mode" is equal to the unconstrained one. This result shows that the latter is physically acceptable.

The difference between the two solutions, "Decreasing IMF mode" and "Standard" or unconstrained mode is illustrated in Fig. 1.

\subsection{The nucleus of the LINER NGC 4278}

As shown in Table 4 Col. 2, the unconstrained model suggests that the optical spectrum of this region is dominated by dwarf stars as well as a metal-rich population. An internal reddening $E(B-V)$ of $\sim 0.02$ is detected.

The solution of the "Decreasing IMF mode" presents a much larger synthetic distance (with values exceeding that of the unconstrained solution by more than $5 \sigma$ ). This shows that this mode is very constraining for the galactic region, a fact that is confirmed through the large discrepancy between the observed and the synthetic spectrum in Fig. 2.

The "Standard mode" provides a solution equivalent to the unconstrained one. It shows a small contribution of G4V stars badly determined suggesting an earlier location of the turnoff. This led us to impose a value on the contribution of this spectral class and to obtain an acceptable solution (shown in the last column of Table 4) presenting an earlier turnoff and satisfying the constraints of this mode.

\subsection{The nucleus of the starburst galaxy NGC 3310}

According to the unconstrained solution, the MS stars and the metallic stars contribute, respectively, to $\sim 94 \%$ and $\sim 68 \%$ of the luminosity in the nucleus of NGC 3310 (Table 5), implying a stellar population dominated by MS and metallic stars. The best defined turnoff is situated in A1-3V but a turnoff in $\mathrm{O} 7-\mathrm{B} 0 \mathrm{~V}$ is possible as well since a small contribution of these stars is present but badly determined. The reddening is very high, in agreement with the location of the turnoff that indicates an important event of star formation and consequently the presence of a large amount of dust in the region. 
Table 3. Results of the spectral synthesis of the globular cluster G170. Each column displays the stellar contributions to luminosity at $\lambda_{0}=$ $5450 \AA$ with their standard deviations for the various solutions (with different modes and types of constraints) as well as for the unconstrained solution. The over-abundant and under-abundant stars are respectively designated by an " $r$ " and a " $w$ " preceding the spectral type and luminosity class. $D^{2}$ is the synthetic distance (or the "mean" residual $E W$ ) with its standard deviation computed in the appendix and $E(B-V)$ is the reddening that can be deduced as described in the text.

\begin{tabular}{|c|c|c|c|c|c|}
\hline Star & $\begin{array}{l}\text { Unconstrained } \\
\text { solution }\end{array}$ & $\begin{array}{c}\text { Mass interval } \\
\text { Dec. IMF mode }\end{array}$ & $\begin{array}{l}\text { Dec. IMF } \\
\text { mode }\end{array}$ & $\begin{array}{l}\text { Mass interval } \\
\text { Standard mode }\end{array}$ & $\begin{array}{c}\text { Standard } \\
\text { mode }\end{array}$ \\
\hline O7-BOV & 0 & $17 M_{\odot}-30 M_{\odot}$ & 0 & $17 M_{\odot}-30 M_{\odot}$ & 0 \\
\hline B3-4V & 0 & $3 M_{\odot}-17 M_{\odot}$ & $0.01 \pm 2$ & $3 M_{\odot}-17 M_{\odot}$ & 0 \\
\hline A1-3V & 0 & $1.6 M_{\odot}-3 M_{\odot}$ & 0 & $1.6 M_{\odot}-3 M_{\odot}$ & 0 \\
\hline $\mathrm{F} 2 \mathrm{~V}$ & 0 & $0.8 M_{\odot}-1.6 M_{\odot}$ & 0 & $1.4 M_{\odot}-1.6 M_{\odot}$ & 0 \\
\hline F8-9V & 0 & $0.8 M_{\odot}-1.6 M_{\odot}$ & 0 & $1.1 M_{\odot}-1.4 M_{\odot}$ & 0 \\
\hline G4V & $22 \pm 11$ & $0.8 M_{\odot}-1.6 M_{\odot}$ & $7 \pm 12$ & $0.8 M_{\odot}-1.1 M_{\odot}$ & $22 \pm 11$ \\
\hline G9-K0V & 0 & $\leq 0.8 M_{\odot}$ & 0 & $0.7 M_{\odot}-0.8 M_{\odot}$ & 0 \\
\hline $\mathrm{K} 5 \mathrm{~V}$ & $27 \pm 7$ & $\leq 0.8 M_{\odot}$ & $11 \pm 5$ & $0.5 M_{\odot}-0.7 M_{\odot}$ & $27 \pm 7$ \\
\hline M2V & 0 & $\leq 0.8 M_{\odot}$ & $2 \pm 1$ & $\leq 0.5 M_{\odot}$ & 0 \\
\hline rG0IV & 0 & $0.8 M_{\odot}-1.6 M_{\odot}$ & 0 & $1.1 M_{\odot}-1.4 M_{\odot}$ & 0 \\
\hline rG5IV & 0 & $0.8 M_{\odot}-1.6 M_{\odot}$ & 0 & $1.1 M_{\odot}-1.4 M_{\odot}$ & 0 \\
\hline $\mathrm{rK} 0 \mathrm{~V}$ & $8 \pm 6$ & $\leq 0.8 M_{\odot}$ & $18 \pm 5.5$ & $0.7 M_{\odot}-0.8 M_{\odot}$ & $8 \pm 6$ \\
\hline $\mathrm{rK} 3 \mathrm{~V}$ & 0 & $\leq 0.8 M_{\odot}$ & 0 & $0.7 M_{\odot}-0.8 M_{\odot}$ & 0 \\
\hline rM1V & 0 & $\leq 0.8 M_{\odot}$ & 0 & $\leq 0.5 M_{\odot}$ & 0 \\
\hline G0-4III & $31 \pm 13$ & $0.8 M_{\odot}-1.6 M_{\odot}$ & $50 \pm 7$ & $1.1 M_{\odot}-1.4 M_{\odot}$ & $31 \pm 13$ \\
\hline wG8III & 0 & $0.8 M_{\odot}-1.6 M_{\odot}$ & $0.01 \pm 6$ & $1.1 M_{\odot}-1.4 M_{\odot}$ & 0 \\
\hline G9III & 0 & $0.8 M_{\odot}-1.6 M_{\odot}$ & 0 & $1.1 M_{\odot}-1.4 M_{\odot}$ & 0 \\
\hline K4III & 0 & $0.8 M_{\odot}-1.6 M_{\odot}$ & 0 & $1.1 M_{\odot}-1.4 M_{\odot}$ & 0 \\
\hline M0.5III & $11 \pm 1$ & $0.8 M_{\odot}-1.6 M_{\odot}$ & $8 \pm 1$ & $1.1 M_{\odot}-1.4 M_{\odot}$ & $11 \pm 1$ \\
\hline M4III & 0 & $0.8 M_{\odot}-1.6 M_{\odot}$ & 0 & $1.4 M_{\odot}-1.6 M_{\odot}$ & 0 \\
\hline M5III & $1 \pm 0.1$ & $0.8 M_{\odot}-1.6 M_{\odot}$ & $1 \pm 0.1$ & $1.4 M_{\odot}-1.6 M_{\odot}$ & $1 \pm 0.1$ \\
\hline rG9III & 0 & $0.8 M_{\odot}-1.6 M_{\odot}$ & 0 & 1. $1 M_{\odot}-1.4 M_{\odot}$ & 0 \\
\hline rK3III & 0 & $0.8 M_{\odot}-1.6 M_{\odot}$ & 0 & $1.1 M_{\odot}-1.4 M_{\odot}$ & 0 \\
\hline rK3IIIbis & 0 & $0.8 M_{\odot}-1.6 M_{\odot}$ & 0 & $1.1 M_{\odot}-1.4 M_{\odot}$ & 0 \\
\hline rK5III & 0 & $0.8 M_{\odot}-1.6 M_{\odot}$ & $2 \pm 2$ & $1.1 M_{\odot}-1.4 M_{\odot}$ & 0 \\
\hline G0Iab & 0 & $3 M_{\odot}-17 M_{\odot}$ & 0 & $3 M_{\odot}-17 M_{\odot}$ & 0 \\
\hline K4Iab & 0 & $3 M_{\odot}-17 M_{\odot}$ & 0 & $3 M_{\odot}-17 M_{\odot}$ & 0 \\
\hline M2Ia & 0 & $17 M_{\odot}-30 M_{\odot}$ & $1 \pm 1$ & $17 M_{\odot}-30 M_{\odot}$ & 0 \\
\hline rG2Iab & 0 & $3 M_{\odot}-17 M_{\odot}$ & 0 & $3 M_{\odot}-17 M_{\odot}$ & 0 \\
\hline rK0II & 0 & $3 M_{\odot}-17 M_{\odot}$ & 0 & $3 M_{\odot}-17 M_{\odot}$ & 0 \\
\hline rK3Iab & 0 & $3 M_{\odot}-17 M_{\odot}$ & 0 & $3 M_{\odot}-17 M_{\odot}$ & 0 \\
\hline$D^{2}$ or $\chi^{2}$ & $7.6 \pm 0.4$ & & $8.0 \pm 0.35$ & & $7.6 \pm 0.4$ \\
\hline$E(B-V)$ & 0.05 & & 0.08 & & 0.05 \\
\hline
\end{tabular}

The solution of the "Decreasing IMF" mode presents a synthetic distance a little more than $\sim 1 \sigma$ higher than that of the unconstrained solution. It may therefore be acceptable (see also Fig. 3). This solution distributes the non zero contributions to a larger number of dwarfs and confirms the high contribution to luminosity of dwarfs and metallic stars (resp. 95\% and 49\%) as well as the location of the turnoff situated in A1-3 V (corresponding to an age of 200 million years for the last burst of star formation). In this solution, the constraint involving the O7-B0V stars is satisfied on the border of the domain of constraints (i.e. with equalities). This suggests that the "Decreasing IMF mode" is very restrictive in this object; the absence of the hot O7-B0V stars might therefore not be real. This conclusion is supported by the presence of emission lines in the spectrum of the starburst galaxy which suggests ongoing star formation in the nucleus of this galaxy. 
G170: Unconstrained solution

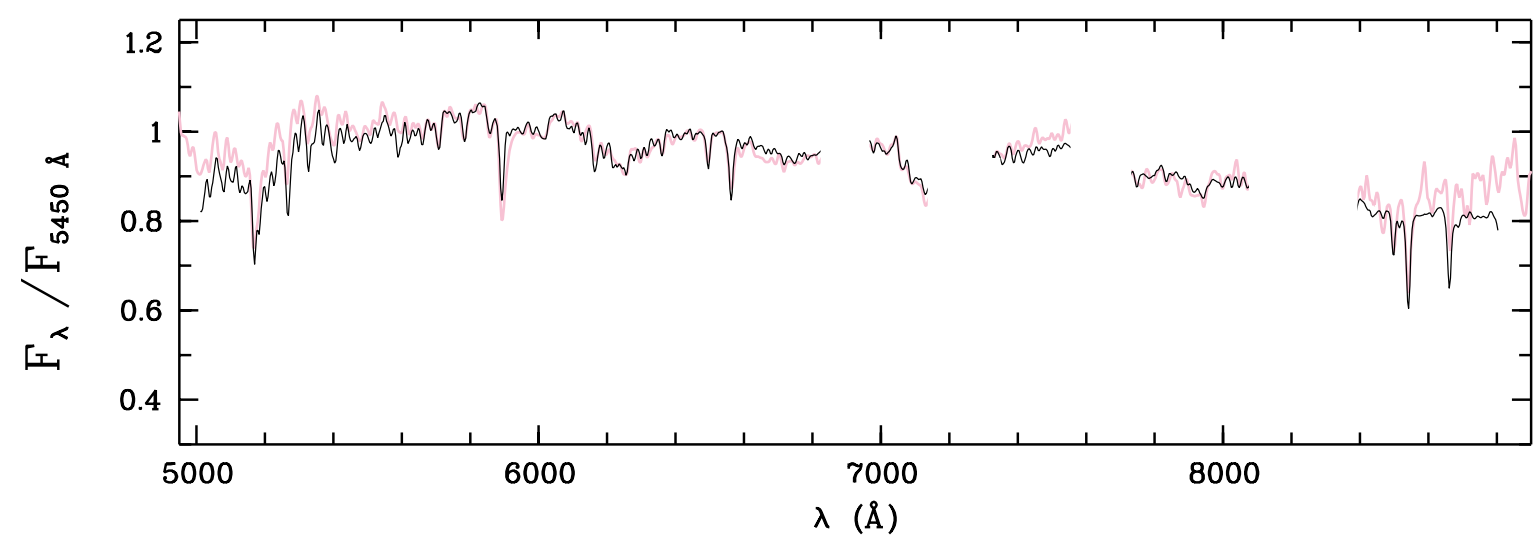

G170: Decreasing IMF mode

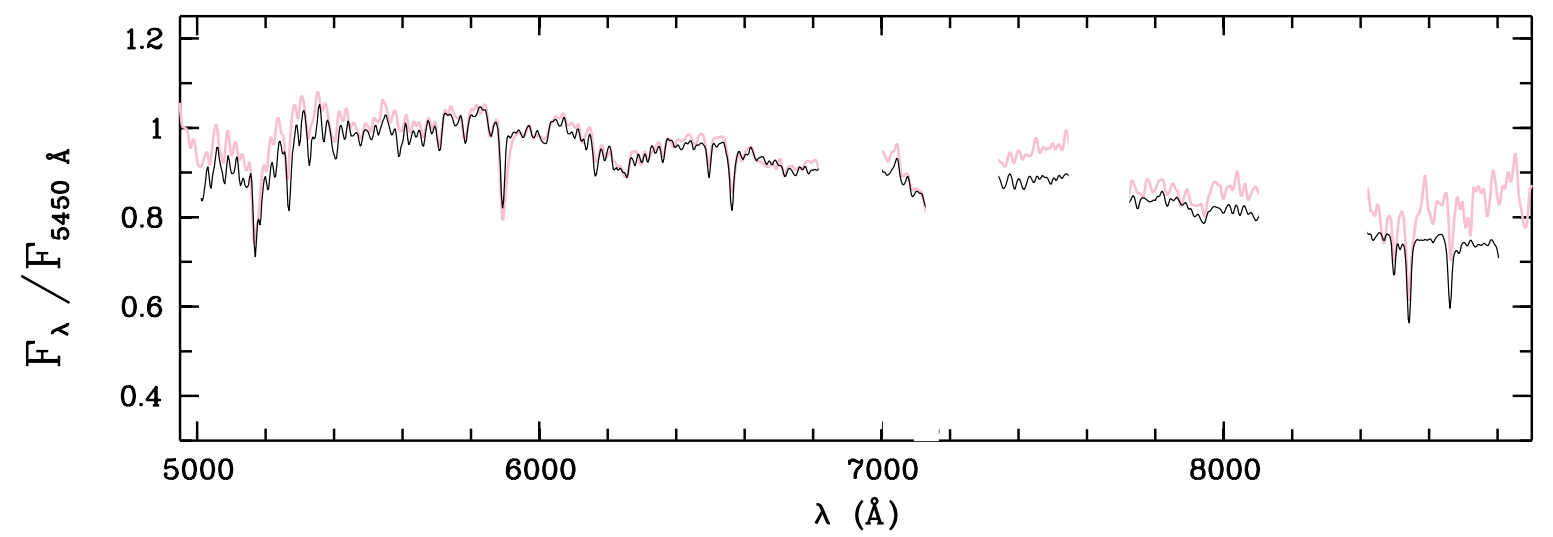

Fig. 1. NGC G170: Synthetic and observed spectra respectively in dark and light lines for different solutions.

The same scenario occurs in the "Standard mode" where the solution provides an acceptable synthetic distance. The best defined turnoff is situated in G5IV but small non-zero contributions (not well defined) show that a turnoff at earlier types is possible. We show in the last column of Table 5 the example of such a situation where we impose the contributions of a B2-3V star in addition to the "Standard mode" constraints. In this solution, the synthetic distance is acceptable which leads us to the same conclusion as previously.

\subsection{The nucleus of the Seyfert 2 galaxy NGC 2110}

The population in the unconstrained solution is dominated by dwarf stars and is moderately metallic ( $\sim 68 \%$ of the optical luminosity is due to MS stars and $\sim 52 \%$ is due to overabundant stars, see Table 6 and Fig. 5). The best defined turnoff is situated in K0V but according to the previous discussion, an earlier turnoff is possible as well.

The solution of the "Decreasing IMF" mode is acceptable since its synthetic distance is slightly higher than that of the unconstrained solution and lies at less than $1 \sigma$ from this one. In this mode, the contribution of dwarf stars is enhanced $(\sim 78 \%)$ while overabundant stars contribute less to the visible luminosity (only $\sim 38 \%$ ). The best defined turnoff is situated in $\mathrm{K} 3 \mathrm{~V}$ but a turnoff in F2V is also possible, a fact that is confirmed in the particular solution where we imposed a contribution of $\sim 3 \%$ to the star class F2V (see also Fig. 5).
In the "Standard mode", the solution presents a synthetic distance equal to that of the unconstrained solution. The solution is very similar to the unconstrained one.

\section{Conclusion}

The ideal case for a spectral synthesis giving a synthetic distance equal to zero would be where the signal to noise ratio of the galactic and stellar spectra goes to infinity and where the stellar database is complete. In such a case, all stars with spectral types later than the spectral type at the turnoff position would have non zero contributions to luminosity. But in practice all unconstrained solutions show many zero contributions; this is due to the finite signal to noise ratio of our spectra and to the limitation of the stellar database which itself is due to the finite spectral resolution.

Therefore, constraining the stellar population would a priori reduce the number of zero contributions because of the additive information introduced in this process. But as can be seen in the previous results, no large improvement in eliminating the zero contribution has appeared. This is probably due to the incomplete set of observational data used for the synthesis. Actually as the constraints are expressed by large inequalities (i.e. equalities are allowed) optima are usually located on the border of the domain in which solutions are constrained.

The stellar synthesis method with constraints presented in this paper has been applied to the 27 regions of galaxies studied 
in Paper IV. In general, all 27 regions present "Standard mode" solutions equal or very similar to the unconstrained solution. Moreover, all zero contributions in the unconstrained solutions remain null in the "Standard mode" or have small ill-defined values and all well- and ill-determined contributions remain as such. This result shows that "Standard mode" solutions are generally included inside the error bars of the unconstrained solution and when they are not, their synthetic distances are at several $\sigma$ from that of the unconstrained solution.

In the "Decreasing IMF mode", the number of star classes contributing to the synthesis is often larger than that of the unconstrained solution and of the "Standard mode". This fact affects especially dwarf stars and is due to the sharper distribution of stars in the mass groups of the H-R diagram in this mode. For the same reason and because the number of constraints is larger, the synthetic distances here are in general larger than those of the previous mode and of the unconstrained solution.

In both modes ("Standard" and "Decreasing IMF") some solutions satisfy their constraints on the border of the domain. This shows that in such cases constraints are too strong and induce bias. However, these solutions provide some indications, thanks to the error bars, allowing one to find acceptable solutions that satisfy the desired conditions inside the domain of constraints (see previous examples).

The solution of the least square problem is the one that minimizes the synthetic distance; this happens often on the border of the domain of constraints but the goal is not to find the optimal mathematical solution, but rather a "realistic" or physical one next to the minimum.

All previous results are confirmed in the case of the globular cluster G170. This is a very important point since this object experiences a single burst of star formation; consequently, any deviation of the behaviour of the resulting stellar population due to the inclusion of astrophysical constraints can clearly be detected.

This study has shown that the inverse method described here and in Papers I-III is very stable against the inclusion of additional astrophysical constraints, and is, therefore, very reliable.
However, constraining the solutions and using the information provided by the error analysis allows one to find similar solutions with younger bursts of star formation. Thus, it is crucial to perform tests such as in the previous section and to discuss the results, especially the different possible locations of the turnoffs, i.e. the age of the last burst of star formation.

Acknowledgements. We would like to thank Pascale Jablonka for kindly providing the spectrum of the globular cluster G170.

\section{References}

Bica, E. 1988, A\&A, 195, 76

Boisson, C., Joly, M., Moultaka, J., Pelat, D., \& Serote Roos, M. 2000, A\&A, 357, 850

Bruzual, G. A., \& Charlot, S. 1993, ApJ, 405, 538

Bruzual, G., \& Charlot, S. 2003, MNRAS, 344, 1000

Charlot, S., \& Bruzual, G. 1991, ApJ, 367, 126

Faber, S. M. 1972, A\&A, 20, 361

Fioc, M., \& Rocca-Volmerange, B. 1997, A\&A, 326, 950

Fluks, M. A., Plez, B., Thé, P. S., et al. 1994, A\&AS, 105, 311

Howarth, I. D. 1983, MNRAS, 203,301

Jablonka, P., Alloin, D., \& Bica, E. 1992, A\&A, 260, 97

Joly, M. 1974, A\&A, 33, 177

Kroupa, P., Tout, C. A., \& Gilmore, G. 1993, MNRAS, 262, 545

Leitherer, C., Schaerer, D., Goldader, J. D., et al. 1999, ApJS, 123, 3

Moultaka, J., \& Pelat, D. 2000, MNRAS, 314, 409

O'Connell, R. W. 1976, ApJ, 206, 390

Pelat, D. 1997, MNRAS, 284, 365

Pelat, D. 1998, MNRAS, 299, 877

Pickles, A. J. 1985, ApJ, 296, 340

Salpeter, E. 1955, ApJ, 121, 161

Scalo, J. M. 1986, Fund. Cos. Phys., 11, 1

Schmidt, A. A., Bica, E., \& Dottori, H. A. 1989, MNRAS, 238, 925

Serote Roos, M., Boisson, C., \& Joly, M. 1996, A\&AS, 117, 93

Schmidt-Kaler, Th. 1982, in Landolt-Börnstein, Stars and star clusters, Numerical data and functional relationships in science and technology, Group IV, Vol. 2b, ed. K. Schaifers, \& H. H. Voight

Silva, D. R. 1991, Ph.D. Thesis, University of Michigan

Silva, D. R., \& Cornell, M. E. 1992, ApJS, 81, 865

Tinsley, B. M. 1972, A\&A, 20, 383

Vazdekis, A. 1999, ApJ, 513, 224 
J. Moultaka et al.: Constraining the solutions of a stellar population synthesis method, Online Material p 1

\section{Online Material}




\section{Appendix A: Calculus of the standard deviation on the synthetic distance}

In this appendix, we compute the standard deviations on the synthetic distance due to observational errors around the studied object. This calculation is complementary to the error analysis made in Paper III where only the standard deviations around the stellar contributions and the variance-covariance matrices were computed. As the synthetic distance is a scalar, its variance-covariance matrix is reduced to its variance.

Thus, here we search for the deviations $\mathrm{d}\left(D^{2}\right)$ around the square of the obtained synthetic distance $D_{0}^{2}$ due to deviations $\mathrm{d} \boldsymbol{W}_{\text {obs }}$ around the observation $\boldsymbol{W}_{\text {obs } 0 \text {. We recall that this }}$ computation is only valuable in the overdetermined case (in the underdetermined case, this distance is equal to zero).

If we make a change of variables on the equivalent widths as $W_{j}^{\prime}=P_{j}^{1 / 2} W_{j}$, the square of the synthetic distance will be written as follows:

$D^{2}=\left(\boldsymbol{W}_{\text {syn }}^{\prime}-\boldsymbol{W}_{\text {obs }}^{\prime}\right)^{T}\left(\boldsymbol{W}_{\text {syn }}^{\prime}-\boldsymbol{W}_{\text {obs }}^{\prime}\right)$.

Then a differenciation around $D_{0}^{2}$ gives:

$\mathrm{d} D^{2}=2\left(\mathrm{~d} \boldsymbol{W}_{\text {syn }}^{\prime}-\mathrm{d} \boldsymbol{W}_{\text {obs }}^{\prime}\right)^{T}\left(\boldsymbol{W}_{\text {syn } 0}^{\prime}-\boldsymbol{W}_{\text {obs } 0}^{\prime}\right)$.

Now, if we replace $\mathrm{d} \boldsymbol{W}_{\text {syn }}^{\prime}$ by $\mathbf{H d} \boldsymbol{W}_{\text {obs }}^{\prime}$, where $\mathbf{H}$ is the orthogonal projector on the synthetic surface (see Paper III), then we get:

$$
\begin{aligned}
\mathrm{d} D^{2} & =2\left((\mathbf{H}-\mathbf{I d}) \mathrm{d} \boldsymbol{W}_{\mathrm{obs}}^{\prime}\right)^{T}\left(\boldsymbol{W}_{\operatorname{syn} 0}^{\prime}-\boldsymbol{W}^{\prime}{ }_{\text {obs } 0}\right) \\
& =2 \mathrm{~d} \boldsymbol{W}^{\prime T}{ }_{\text {obs }}\left(\mathbf{H}^{T}-\mathbf{I d}\right)\left(\boldsymbol{W}_{\operatorname{syn} 0}^{\prime}-\boldsymbol{W}^{\prime}{ }_{\text {obs } 0}\right) .
\end{aligned}
$$

where Id is the identity matrix.

Let us write, on the one hand, the definition of the variance of the quantity $\mathrm{d} D^{2}$ :

$\operatorname{var}\left(\mathrm{d} D^{2}\right)=\left\langle\mathrm{d} D^{2} \mathrm{~d} D^{2 T}\right\rangle-\left\langle\mathrm{d} D^{2}\right\rangle^{2}$

on the other hand, we have $\left\langle\mathrm{d} D^{2}\right\rangle=2 D_{0}\langle\mathrm{~d} D\rangle$. Then if we translate the origin of the vector space in $\boldsymbol{W}^{\prime}$ syn0, we can consider the subspace of dimension 1 of which the generator vector is $\boldsymbol{W}^{\prime}{ }_{\text {obs } 0}-\boldsymbol{W}_{\text {syn } 0}^{\prime}$. In this subspace the synthetic distance is described by the same vector $\boldsymbol{D}_{0}=\boldsymbol{W}^{\prime}{ }_{\text {obs } 0}-\boldsymbol{W}_{\text {syn } 0}^{\prime}$ and the deviation to this distance is vector $\mathrm{d} \boldsymbol{D}=\boldsymbol{D}-\boldsymbol{D}_{0}$. Thus, if we call $\boldsymbol{u}$ the unit vector of this subspace $\left(\boldsymbol{u}=\frac{\boldsymbol{W}_{\text {obs } 0-\boldsymbol{W}^{\prime}{ }_{\text {syn } 0}}}{\left\|\boldsymbol{W}_{\text {obs } 0}^{\prime}-\boldsymbol{W}_{\text {syn } 0}^{\prime}\right\|}\right.$, we can construct the orthogonal projector over it as $\mathbf{P}=\boldsymbol{u} \boldsymbol{u}^{T}$; then we get $\mathrm{d} \boldsymbol{D}=\mathbf{P} \mathrm{d} \boldsymbol{W}^{\prime}{ }_{\text {obs }}$ and $\langle\mathrm{d} D\rangle=\langle\mathrm{d} \boldsymbol{D}\rangle=\mathbf{P}\left\langle\mathrm{d} \boldsymbol{W}^{\prime}{ }_{\text {syn }}\right\rangle=0$. This implies that $\left\langle\mathrm{d} D^{2}\right\rangle=0$ and $\operatorname{var}\left(\mathrm{d} D^{2}\right)=\operatorname{var}\left(D^{2}\right)$.

Back to Eq. (A.4), we have:

$$
\begin{aligned}
\operatorname{var}\left(\mathrm{d} D^{2}\right)= & \operatorname{var}\left(D^{2}\right) \\
= & \left\langle\mathrm{d} D^{2} \mathrm{~d} D^{2}\right\rangle \\
= & 4 \Delta \boldsymbol{W}^{\prime T}(\mathbf{H}-\mathbf{I d})\left\langle\mathrm{d} \boldsymbol{W}^{\prime}{ }_{\mathrm{obs}} \mathrm{d} \boldsymbol{W}^{\prime T}{ }_{\mathrm{obs}}\right\rangle \\
& \times\left(\mathbf{H}^{T}-\mathbf{I d}\right) \Delta \boldsymbol{W}^{\prime}
\end{aligned}
$$

where $\Delta \boldsymbol{W}^{\prime}=\boldsymbol{W}^{\prime}{ }_{\text {obs } 0}-\boldsymbol{W}^{\prime}{ }_{\text {syn } 0}$. Then

$\sigma_{D^{2}}=2 \sqrt{\Delta \boldsymbol{W}^{\prime T}(\mathbf{H}-\mathbf{I d}) \mathbf{V}^{\prime}{ }_{\mathrm{obs}}\left(\mathbf{H}^{T}-\mathbf{I d}\right) \Delta \boldsymbol{W}^{\prime}}$.

In addition, as $\langle\mathrm{d} D\rangle=\left\langle\mathrm{d} D^{2}\right\rangle=0$, we set the simple equation

$\sigma_{D}=\frac{\sigma_{D^{2}}}{2 D_{0}}$. 
J. Moultaka et al.: Constraining the solutions of a stellar population synthesis method, Online Material p 3

NGC 4278: Unconstrained solution
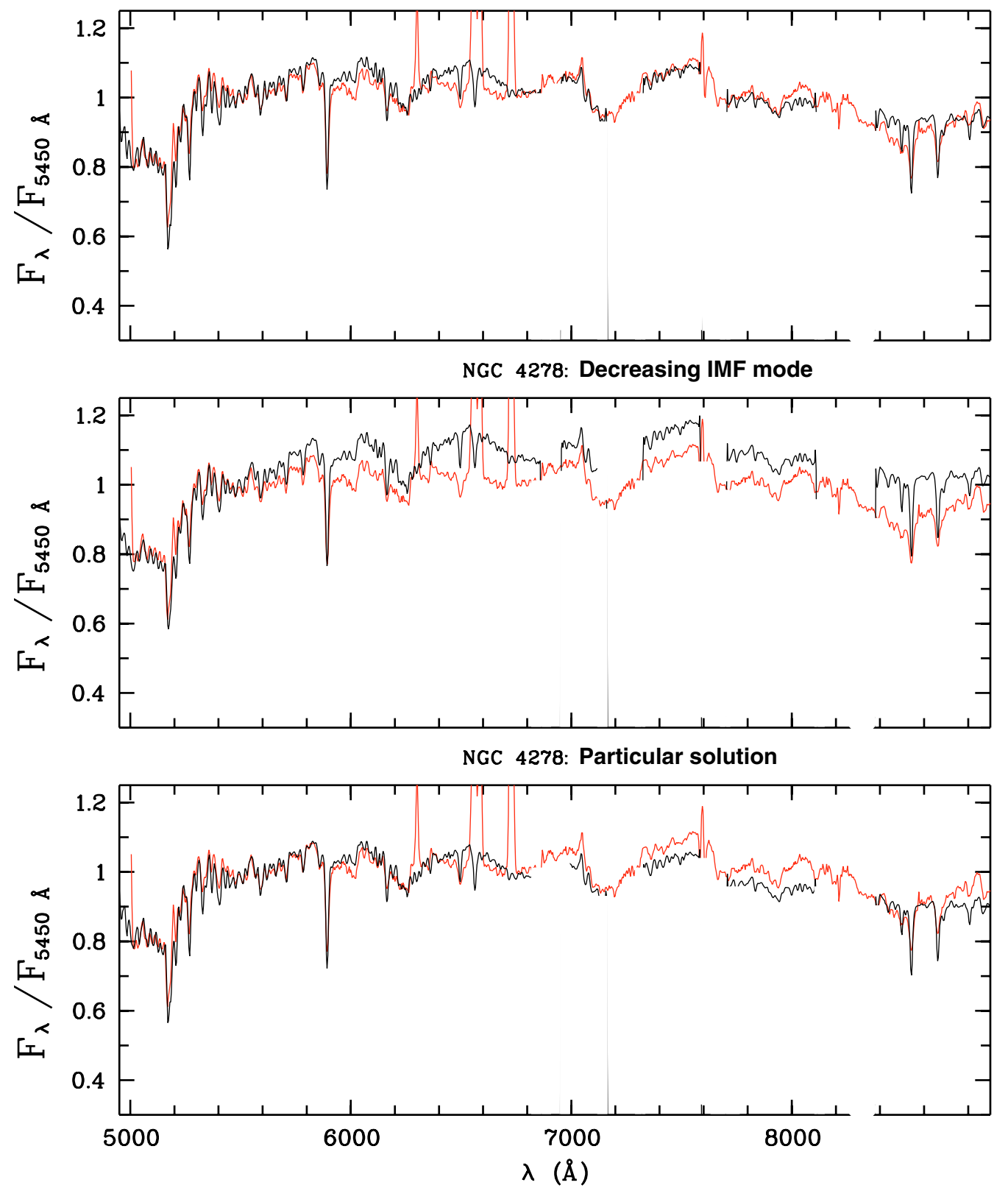

Fig. 2. NGC 4278: same as Fig. 1. 
J. Moultaka et al.: Constraining the solutions of a stellar population synthesis method, Online Material p 4

NGC 3310: Unconstrained solution
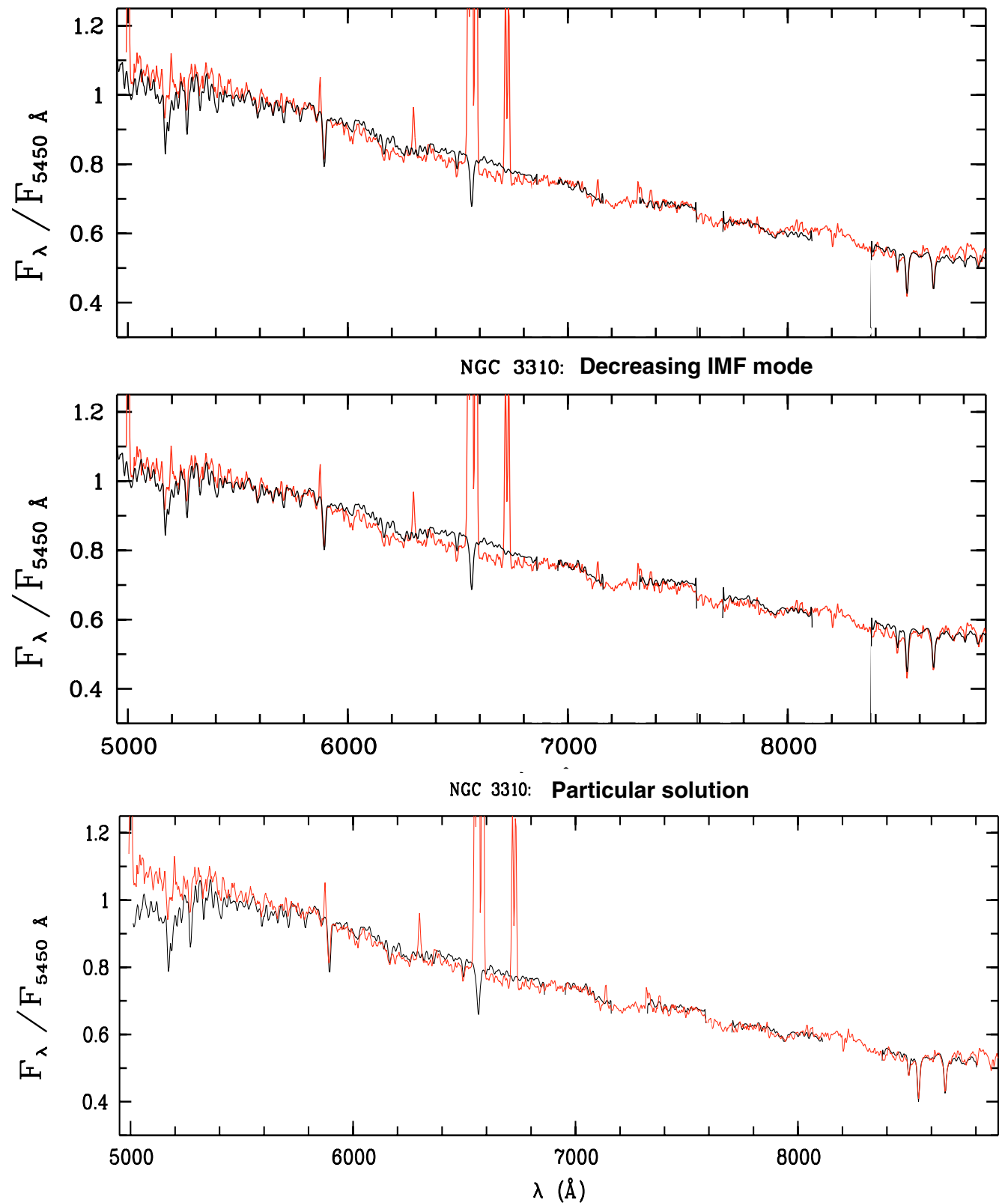

Fig. 3. NGC 3310: same as Fig. 1. 
J. Moultaka et al.: Constraining the solutions of a stellar population synthesis method, Online Material p 5

NGC 2110: Unconstrained solution
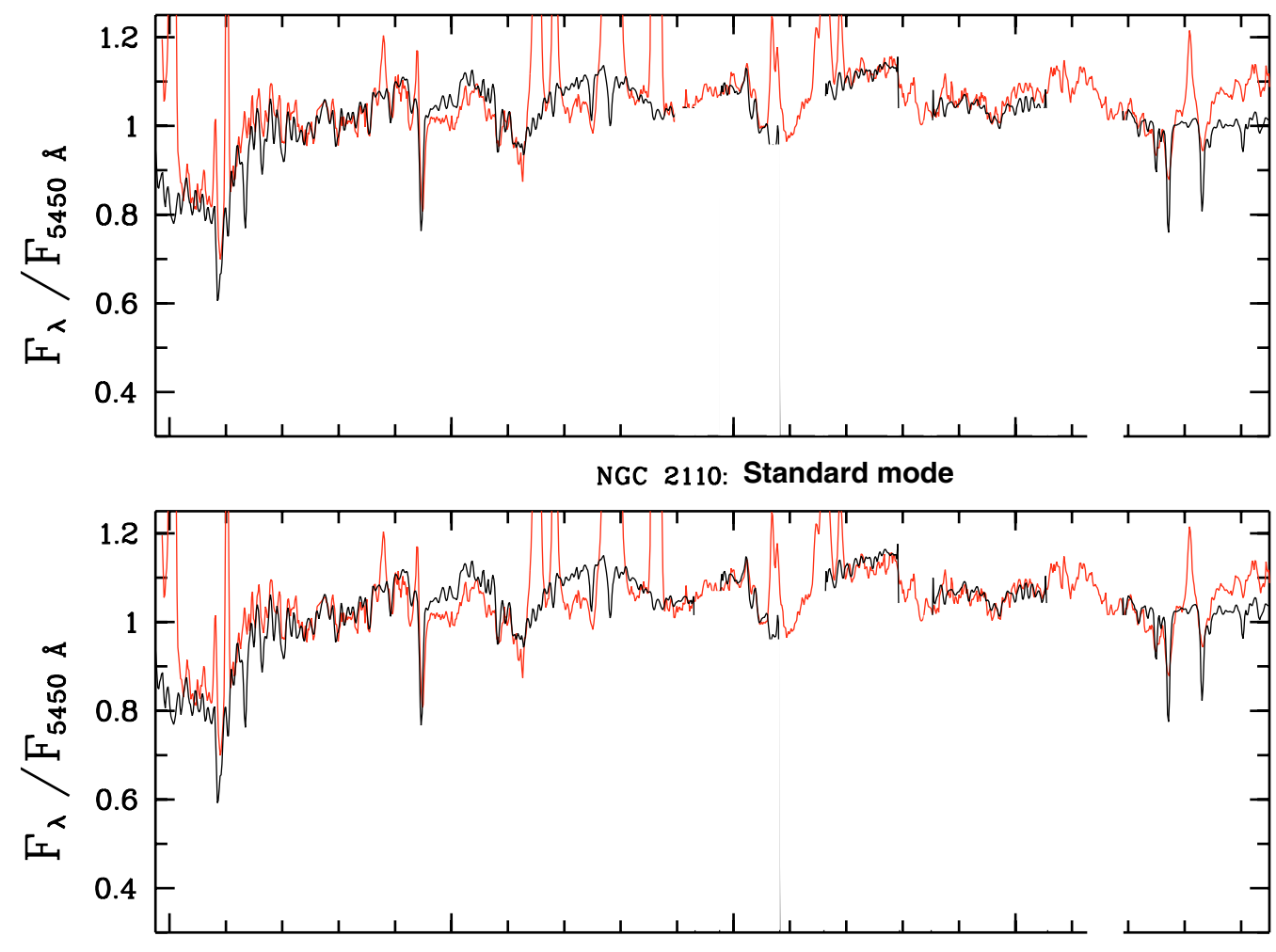

NGC 2110: Decreasing IMF mode

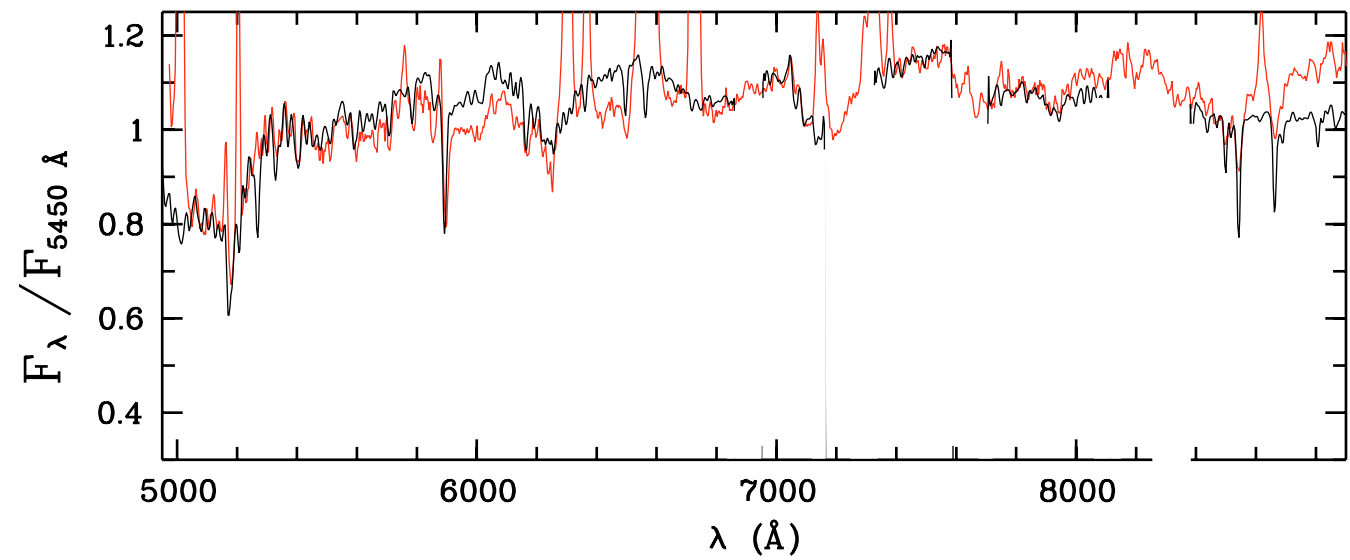

Fig. 4. NGC 2110: same as Fig. 1. 
J. Moultaka et al.: Constraining the solutions of a stellar population synthesis method, Online Material p 6 NGC 2110: Particular solution

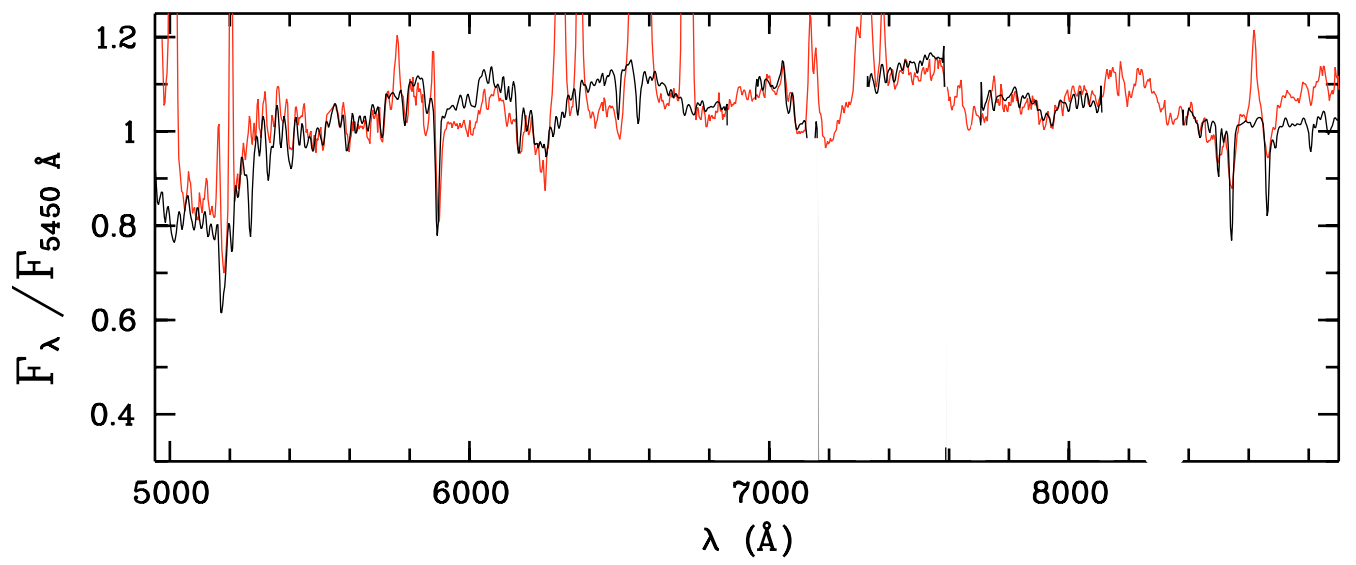

Fig. 5. NGC 2110: particular solution. Same as Fig. 1. 
J. Moultaka et al.: Constraining the solutions of a stellar population synthesis method, Online Material $p 7$

Table 4. The different synthesis solutions for the nucleus of NGC 4278. Same notation as in Table 3. The underlined contribution in the particular solution is imposed. In the reddening row, $\mathrm{R}$ means that the synthetic spectrum is redder than the observed spectrum.

\begin{tabular}{|c|c|c|c|c|c|c|}
\hline Star & $\begin{array}{c}\text { Unconstrained } \\
\text { solution }\end{array}$ & $\begin{array}{c}\text { Mass interval } \\
\text { Dec. IMF mode }\end{array}$ & $\begin{array}{l}\text { Dec. IMF } \\
\text { mode }\end{array}$ & $\begin{array}{c}\text { Mass interval } \\
\text { Standard mode }\end{array}$ & $\begin{array}{c}\text { Standard } \\
\text { mode }\end{array}$ & $\begin{array}{c}\text { Particular } \\
\text { solution }\end{array}$ \\
\hline O7-BOV & 0 & $17 M_{\odot}-30 M_{\odot}$ & 0 & $17 M_{\odot}-30 M_{\odot}$ & 0 & 0 \\
\hline B3-4V & 0 & $3 M_{\odot}-17 M_{\odot}$ & 0 & $3 M_{\odot}-17 M_{\odot}$ & 0 & 0 \\
\hline A1-3V & 0 & $1.6 M_{\odot}-3 M_{\odot}$ & 0 & $1.6 M_{\odot}-3 M_{\odot}$ & 0 & 0 \\
\hline F2V & 0 & $0.8 M_{\odot}-1.6 M_{\odot}$ & 0 & $1.4 M_{\odot}-1.6 M_{\odot}$ & 0 & 0 \\
\hline F8-9V & 0 & $0.8 M_{\odot}-1.6 M_{\odot}$ & 0 & $1.1 M_{\odot}-1.4 M_{\odot}$ & 0 & 0 \\
\hline G4V & 0 & $0.8 M_{\odot}-1.6 M_{\odot}$ & $1 \pm 2$ & $0.8 M_{\odot}-1.1 M_{\odot}$ & $0.2 \pm 1$ & $\underline{7}$ \\
\hline G9-K0V & 0 & $\leq 0.8 M_{\odot}$ & 0 & $0.7 M_{\odot}-0.8 M_{\odot}$ & 0 & 0 \\
\hline K5V & 0 & $\leq 0.8 M_{\odot}$ & $42 \pm 4$ & $0.5 M_{\odot}-0.7 M_{\odot}$ & 0 & 0 \\
\hline M2V & 0 & $\leq 0.8 M_{\odot}$ & $4 \pm 2$ & $\leq 0.5 M_{\odot}$ & 0 & 0 \\
\hline rG0IV & 0 & $0.8 M_{\odot}-1.6 M_{\odot}$ & 0 & $1.1 M_{\odot}-1.4 M_{\odot}$ & 0 & 0 \\
\hline rG5IV & 0 & $0.8 M_{\odot}-1.6 M_{\odot}$ & 0 & $1.1 M_{\odot}-1.4 M_{\odot}$ & 0 & 0 \\
\hline rK0V & $38 \pm 14$ & $\leq 0.8 M_{\odot}$ & 0 & $0.7 M_{\odot}-0.8 M_{\odot}$ & $37 \pm 15$ & $26 \pm 15$ \\
\hline rK3V & $38 \pm 8$ & $\leq 0.8 M_{\odot}$ & $35 \pm 3$ & $0.7 M_{\odot}-0.8 M_{\odot}$ & $38 \pm 8$ & $40 \pm 5$ \\
\hline rM1V & $2 \pm 2$ & $\leq 0.8 M_{\odot}$ & $5 \pm 4$ & $\leq 0.5 M_{\odot}$ & $2.5 \pm 2$ & $3 \pm 2$ \\
\hline G0-4III & 0 & $0.8 M_{\odot}-1.6 M_{\odot}$ & 0 & $1.1 M_{\odot}-1.4 M_{\odot}$ & 0 & 0 \\
\hline wG8III & 0 & $0.8 M_{\odot}-1.6 M_{\odot}$ & 0 & $1.1 M_{\odot}-1.4 M_{\odot}$ & 0 & 0 \\
\hline G9III & 0 & $0.8 M_{\odot}-1.6 M_{\odot}$ & 0 & $1.1 M_{\odot}-1.4 M_{\odot}$ & 0 & 0 \\
\hline K4III & 0 & $0.8 M_{\odot}-1.6 M_{\odot}$ & 0 & $1.1 M_{\odot}-1.4 M_{\odot}$ & 0 & 0 \\
\hline M0.5III & $5 \pm 2$ & $0.8 M_{\odot}-1.6 M_{\odot}$ & 0 & $1.1 M_{\odot}-1.4 M_{\odot}$ & $5 \pm 2$ & $4 \pm 1$ \\
\hline M4III & $3.5 \pm 0.1$ & $0.8 M_{\odot}-1.6 M_{\odot}$ & $3 \pm 0.2$ & $1.4 M_{\odot}-1.6 M_{\odot}$ & $3.5 \pm 0.1$ & $3 \pm 0.3$ \\
\hline M5III & 0 & $0.8 M_{\odot}-1.6 M_{\odot}$ & 0 & $1.4 M_{\odot}-1.6 M_{\odot}$ & 0 & 0 \\
\hline rG9III & 0 & $0.8 M_{\odot}-1.6 M_{\odot}$ & 0 & $1.1 M_{\odot}-1.4 M_{\odot}$ & 0 & 0 \\
\hline rK3III & $3 \pm 6$ & $0.8 M_{\odot}-1.6 M_{\odot}$ & 0 & $1.1 M_{\odot}-1.4 M_{\odot}$ & $3 \pm 7$ & $4 \pm 6$ \\
\hline rK3IIIbis & 0 & $0.8 M_{\odot}-1.6 M_{\odot}$ & 0 & $1.1 M_{\odot}-1.4 M_{\odot}$ & 0 & 0 \\
\hline rK5III & $10 \pm 4$ & $0.8 M_{\odot}-1.6 M_{\odot}$ & $10 \pm 2$ & $1.1 M_{\odot}-1.4 M_{\odot}$ & $10 \pm 4$ & $11 \pm 4$ \\
\hline GOIab & 0 & $3 M_{\odot}-17 M_{\odot}$ & 0 & $3 M_{\odot}-17 M_{\odot}$ & 0 & 0 \\
\hline K4Iab & 0 & $3 M_{\odot}-17 M_{\odot}$ & 0 & $3 M_{\odot}-17 M_{\odot}$ & 0 & 0 \\
\hline M2Ia & 0 & $17 M_{\odot}-30 M_{\odot}$ & 0 & $17 M_{\odot}-30 M_{\odot}$ & 0 & 0 \\
\hline rG2Iab & 0 & $3 M_{\odot}-17 M_{\odot}$ & 0 & $3 M_{\odot}-17 M_{\odot}$ & 0 & 0 \\
\hline rK0II & 0 & $3 M_{\odot}-17 M_{\odot}$ & 0 & $3 M_{\odot}-17 M_{\odot}$ & 0 & 0 \\
\hline rK3Iab & 0 & $3 M_{\odot}-17 M_{\odot}$ & 0 & $3 M_{\odot}-17 M_{\odot}$ & 0 & 0 \\
\hline$D^{2}$ or $\chi^{2}$ & $12.3 \pm 0.95$ & & $17.1 \pm 1.6$ & & $12.3 \pm 0.9$ & $13.0 \pm 1.2$ \\
\hline$E(B-V)$ & 0.02 & & $0.00 \mathrm{R}$ & & 0.02 & 0.00 \\
\hline
\end{tabular}


J. Moultaka et al.: Constraining the solutions of a stellar population synthesis method, Online Material p 8

Table 5. Results of the spectral synthesis of NGC 3310 nucleus. Same notation as in Tables 3 and 4.

\begin{tabular}{|c|c|c|c|c|c|c|}
\hline Star & $\begin{array}{c}\text { Unconstrained } \\
\text { solution }\end{array}$ & $\begin{array}{c}\text { Mass interval } \\
\text { Dec. IMF mode }\end{array}$ & $\begin{array}{l}\text { Dec. IMF } \\
\text { mode }\end{array}$ & $\begin{array}{l}\text { Mass interval } \\
\text { Standard mode }\end{array}$ & $\begin{array}{l}\text { Standard } \\
\text { mode }\end{array}$ & $\begin{array}{l}\text { Particular } \\
\text { solution }\end{array}$ \\
\hline O7-BOV & $4 \pm 5$ & $17 M_{\odot}-30 M_{\odot}$ & 0 & $17 M_{\odot}-30 M_{\odot}$ & $4 \pm 33$ & 0 \\
\hline B3-4V & 0 & $3 M_{\odot}-17 M_{\odot}$ & 0 & $3 M_{\odot}-17 M_{\odot}$ & $0.1 \pm 44.5$ & $\underline{10}$ \\
\hline A1-3V & $21 \pm 9$ & $1.6 M_{\odot}-3 M_{\odot}$ & $30 \pm 12.5$ & $1.6 M_{\odot}-3 M_{\odot}$ & $22 \pm 26$ & $15 \pm 9$ \\
\hline $\mathrm{F} 2 \mathrm{~V}$ & 0 & $0.8 M_{\odot}-1.6 M_{\odot}$ & $1 \pm 17$ & $1.4 M_{\odot}-1.6 M_{\odot}$ & 0 & 0 \\
\hline F8-9V & $6 \pm 6$ & $0.8 M_{\odot}-1.6 M_{\odot}$ & 0 & $1.1 M_{\odot}-1.4 M_{\odot}$ & $6 \pm 9$ & $6.5 \pm 6$ \\
\hline G4V & 0 & $0.8 M_{\odot}-1.6 M_{\odot}$ & $6 \pm 11$ & $0.8 M_{\odot}-1.1 M_{\odot}$ & 0 & 0 \\
\hline G9-K0V & 0 & $\leq 0.8 M_{\odot}$ & 0 & $0.7 M_{\odot}-0.8 M_{\odot}$ & 0 & 0 \\
\hline K5V & 0 & $\leq 0.8 M_{\odot}$ & $11 \pm 4$ & $0.5 M_{\odot}-0.7 M_{\odot}$ & 0 & 0 \\
\hline M2V & 0 & $\leq 0.8 M_{\odot}$ & $2 \pm 1$ & $\leq 0.5 M_{\odot}$ & 0 & 0 \\
\hline rG0IV & 0 & $0.8 M_{\odot}-1.6 M_{\odot}$ & 0 & $1.1 M_{\odot}-1.4 M_{\odot}$ & 0 & 0 \\
\hline rG5IV & $30 \pm 10.5$ & $0.8 M_{\odot}-1.6 M_{\odot}$ & $27 \pm 9$ & $1.1 M_{\odot}-1.4 M_{\odot}$ & $30 \pm 10$ & $29 \pm 10$ \\
\hline $\mathrm{rK} 0 \mathrm{~V}$ & $33 \pm 5.5$ & $\leq 0.8 M_{\odot}$ & $18 \pm 7$ & $0.7 M_{\odot}-0.8 M_{\odot}$ & $33 \pm 4.5$ & $34 \pm 5$ \\
\hline $\mathrm{rK} 3 \mathrm{~V}$ & 0 & $\leq 0.8 M_{\odot}$ & 0 & $0.7 M_{\odot}-0.8 M_{\odot}$ & 0 & 0 \\
\hline $\mathrm{rM} 1 \mathrm{~V}$ & 0 & $\leq 0.8 M_{\odot}$ & 0 & $\leq 0.5 M_{\odot}$ & 0 & 0 \\
\hline G0-4III & 0 & $0.8 M_{\odot}-1.6 M_{\odot}$ & 0 & $1.1 M_{\odot}-1.4 M_{\odot}$ & 0 & 0 \\
\hline wG8III & 0 & $0.8 M_{\odot}-1.6 M_{\odot}$ & 0 & $1.1 M_{\odot}-1.4 M_{\odot}$ & 0 & 0 \\
\hline G9III & 0 & $0.8 M_{\odot}-1.6 M_{\odot}$ & 0 & $1.1 M_{\odot}-1.4 M_{\odot}$ & 0 & 0 \\
\hline K4III & 0 & $0.8 M_{\odot}-1.6 M_{\odot}$ & 0 & $1.1 M_{\odot}-1.4 M_{\odot}$ & 0 & 0 \\
\hline M0.5III & 0 & $0.8 M_{\odot}-1.6 M_{\odot}$ & 0 & $1.1 M_{\odot}-1.4 M_{\odot}$ & 0 & 0 \\
\hline M4III & $1 \pm 0.2$ & $0.8 M_{\odot}-1.6 M_{\odot}$ & $1 \pm 0.2$ & $1.4 M_{\odot}-1.6 M_{\odot}$ & $1 \pm 0.2$ & $1 \pm 0.2$ \\
\hline M5III & 0 & $0.8 M_{\odot}-1.6 M_{\odot}$ & 0 & $1.4 M_{\odot}-1.6 M_{\odot}$ & 0 & 0 \\
\hline rG9III & 0 & $0.8 M_{\odot}-1.6 M_{\odot}$ & 0 & $1.1 M_{\odot}-1.4 M_{\odot}$ & 0 & 0 \\
\hline rK3III & 0 & $0.8 M_{\odot}-1.6 M_{\odot}$ & 0 & $1.1 M_{\odot}-1.4 M_{\odot}$ & 0 & 0 \\
\hline rK3IIIbis & 0 & $0.8 M_{\odot}-1.6 M_{\odot}$ & 0 & $1.1 M_{\odot}-1.4 M_{\odot}$ & 0 & 0 \\
\hline rK5III & $5 \pm 2$ & $0.8 M_{\odot}-1.6 M_{\odot}$ & $4 \pm 2$ & $1.1 M_{\odot}-1.4 M_{\odot}$ & $5 \pm 2$ & $5 \pm 2$ \\
\hline G0Iab & 0 & $3 M_{\odot}-17 M_{\odot}$ & 0 & $3 M_{\odot}-17 M_{\odot}$ & 0 & 0 \\
\hline K4Iab & 0 & $3 M_{\odot}-17 M_{\odot}$ & 0 & $3 M_{\odot}-17 M_{\odot}$ & 0 & 0 \\
\hline M2Ia & 0 & $17 M_{\odot}-30 M_{\odot}$ & 0 & $17 M_{\odot}-30 M_{\odot}$ & 0 & 0 \\
\hline rG2Iab & 0 & $3 M_{\odot}-17 M_{\odot}$ & 0 & $3 M_{\odot}-17 M_{\odot}$ & 0 & 0 \\
\hline rK0II & 0 & $3 M_{\odot}-17 M_{\odot}$ & 0 & $3 M_{\odot}-17 M_{\odot}$ & 0 & 0 \\
\hline rK3Iab & 0 & $3 M_{\odot}-17 M_{\odot}$ & 0 & $3 M_{\odot}-17 M_{\odot}$ & 0 & 0 \\
\hline$D^{2}$ or $\chi^{2}$ & $11.4 \pm 1.5$ & & $13.2 \pm 1.5$ & & $11.4 \pm 1.2$ & $11.6 \pm 1.5$ \\
\hline$E(B-V)$ & 0.23 & & 0.2 & & 0.23 & 0.23 \\
\hline
\end{tabular}


J. Moultaka et al.: Constraining the solutions of a stellar population synthesis method, Online Material $p 9$

Table 6. Results of the spectral synthesis of NGC 2110 nucleus. Same notation as in Tables 3 and 4.

\begin{tabular}{|c|c|c|c|c|c|c|}
\hline Star & $\begin{array}{c}\text { Unconstrained } \\
\text { solution }\end{array}$ & $\begin{array}{c}\text { Mass interval } \\
\text { Dec. IMF mode }\end{array}$ & $\begin{array}{l}\text { Dec. IMF } \\
\text { mode }\end{array}$ & $\begin{array}{l}\text { Mass interval } \\
\text { Standard mode }\end{array}$ & $\begin{array}{l}\text { Standard } \\
\text { mode }\end{array}$ & $\begin{array}{c}\text { Particular } \\
\text { solution }\end{array}$ \\
\hline O7-BOV & $3 \pm 7$ & $17 M_{\odot}-30 M_{\odot}$ & 0 & $17 M_{\odot}-30 M_{\odot}$ & $2 \pm 67$ & 0 \\
\hline B3-4V & 0 & $3 M_{\odot}-17 M_{\odot}$ & 0 & $3 M_{\odot}-17 M_{\odot}$ & $0.1 \pm 71$ & 0 \\
\hline A1-3V & 0 & $1.6 M_{\odot}-3 M_{\odot}$ & 0 & $1.6 M_{\odot}-3 M_{\odot}$ & 0 & 0 \\
\hline $\mathrm{F} 2 \mathrm{~V}$ & 0 & $0.8 M_{\odot}-1.6 M_{\odot}$ & $0.006 \pm 7$ & $1.4 M_{\odot}-1.6 M_{\odot}$ & 0 & $\underline{3}$ \\
\hline F8-9V & 0 & $0.8 M_{\odot}-1.6 M_{\odot}$ & 0 & $1.1 M_{\odot}-1.4 M_{\odot}$ & 0 & 0 \\
\hline G4V & 0 & $0.8 M_{\odot}-1.6 M_{\odot}$ & $3 \pm 18$ & $0.8 M_{\odot}-1.1 M_{\odot}$ & 0 & $2 \pm 14$ \\
\hline G9-K0V & 0 & $\leq 0.8 M_{\odot}$ & 0 & $0.7 M_{\odot}-0.8 M_{\odot}$ & 0 & 0 \\
\hline K5V & $9 \pm 8$ & $\leq 0.8 M_{\odot}$ & $32 \pm 6.5$ & $0.5 M_{\odot}-0.7 M_{\odot}$ & $9 \pm 10$ & $32 \pm 7$ \\
\hline M2V & $6 \pm 2$ & $\leq 0.8 M_{\odot}$ & $6 \pm 1$ & $\leq 0.5 M_{\odot}$ & $6 \pm 1$ & $6 \pm 1$ \\
\hline rG0IV & 0 & $0.8 M_{\odot}-1.6 M_{\odot}$ & 0 & $1.1 M_{\odot}-1.4 M_{\odot}$ & 0 & 0 \\
\hline rG5IV & $9 \pm 12$ & $0.8 M_{\odot}-1.6 M_{\odot}$ & $10 \pm 16$ & $1.1 M_{\odot}-1.4 M_{\odot}$ & $10 \pm 12$ & $9 \pm 15$ \\
\hline $\mathrm{rK} 0 \mathrm{~V}$ & $19 \pm 6$ & $\leq 0.8 M_{\odot}$ & 0 & $0.7 M_{\odot}-0.8 M_{\odot}$ & $19 \pm 8$ & 0 \\
\hline $\mathrm{rK} 3 \mathrm{~V}$ & $22 \pm 5$ & $\leq 0.8 M_{\odot}$ & $27 \pm 3$ & $0.7 M_{\odot}-0.8 M_{\odot}$ & $22 \pm 7$ & $26 \pm 2$ \\
\hline $\mathrm{rM} 1 \mathrm{~V}$ & 0 & $\leq 0.8 M_{\odot}$ & 0 & $\leq 0.5 M_{\odot}$ & 0 & 0 \\
\hline G0-4III & 0 & $0.8 M_{\odot}-1.6 M_{\odot}$ & 0 & $1.1 M_{\odot}-1.4 M_{\odot}$ & 0 & 0 \\
\hline wG8III & 0 & $0.8 M_{\odot}-1.6 M_{\odot}$ & 0 & $1.1 M_{\odot}-1.4 M_{\odot}$ & 0 & 0 \\
\hline G9III & 0 & $0.8 M_{\odot}-1.6 M_{\odot}$ & 0 & $1.1 M_{\odot}-1.4 M_{\odot}$ & 0 & 0 \\
\hline K4III & $15 \pm 5$ & $0.8 M_{\odot}-1.6 M_{\odot}$ & $6 \pm 4$ & $1.1 M_{\odot}-1.4 M_{\odot}$ & $15 \pm 7$ & $6 \pm 4$ \\
\hline M0.5III & $12 \pm 3$ & $0.8 M_{\odot}-1.6 M_{\odot}$ & $12 \pm 3$ & $1.1 M_{\odot}-1.4 M_{\odot}$ & $12 \pm 1.5$ & $11.5 \pm 3$ \\
\hline M4III & $2 \pm 0.3$ & $0.8 M_{\odot}-1.6 M_{\odot}$ & $2 \pm 0.2$ & $1.4 M_{\odot}-1.6 M_{\odot}$ & $2 \pm 0.1$ & $2 \pm 0.2$ \\
\hline M5III & 0 & $0.8 M_{\odot}-1.6 M_{\odot}$ & 0 & $1.4 M_{\odot}-1.6 M_{\odot}$ & 0 & 0 \\
\hline rG9III & 0 & $0.8 M_{\odot}-1.6 M_{\odot}$ & 0 & $1.1 M_{\odot}-1.4 M_{\odot}$ & 0 & 0 \\
\hline rK3III & 0 & $0.8 M_{\odot}-1.6 M_{\odot}$ & 0 & $1.1 M_{\odot}-1.4 M_{\odot}$ & 0 & 0 \\
\hline rK3IIIbis & 0 & $0.8 M_{\odot}-1.6 M_{\odot}$ & 0 & $1.1 M_{\odot}-1.4 M_{\odot}$ & 0 & 0 \\
\hline rK5III & $2.5 \pm 7$ & $0.8 M_{\odot}-1.6 M_{\odot}$ & $1 \pm 8$ & $1.1 M_{\odot}-1.4 M_{\odot}$ & $2.5 \pm 4$ & $2 \pm 8$ \\
\hline G0Iab & 0 & $3 M_{\odot}-17 M_{\odot}$ & 0 & $3 M_{\odot}-17 M_{\odot}$ & 0 & 0 \\
\hline K4Iab & 0 & $3 M_{\odot}-17 M_{\odot}$ & 0 & $3 M_{\odot}-17 M_{\odot}$ & 0 & 0 \\
\hline M2Ia & 0 & $17 M_{\odot}-30 M_{\odot}$ & 0 & $17 M_{\odot}-30 M_{\odot}$ & 0 & 0 \\
\hline rG2Iab & 0 & $3 M_{\odot}-17 M_{\odot}$ & 0 & $3 M_{\odot}-17 M_{\odot}$ & 0 & 0 \\
\hline rK0II & 0 & $3 M_{\odot}-17 M_{\odot}$ & 0 & $3 M_{\odot}-17 M_{\odot}$ & 0 & 0 \\
\hline rK3Iab & 0 & $3 M_{\odot}-17 M_{\odot}$ & 0 & $3 M_{\odot}-17 M_{\odot}$ & 0 & 0 \\
\hline$D^{2}$ or $\chi^{2}$ & $13.1 \pm 2.0$ & & $13.5 \pm 1.9$ & & $13.1 \pm 1.2$ & $13.5 \pm 1.9$ \\
\hline$E(B-V)$ & 0.05 & & 0.0 & & 0.05 & 0.05 \\
\hline
\end{tabular}

